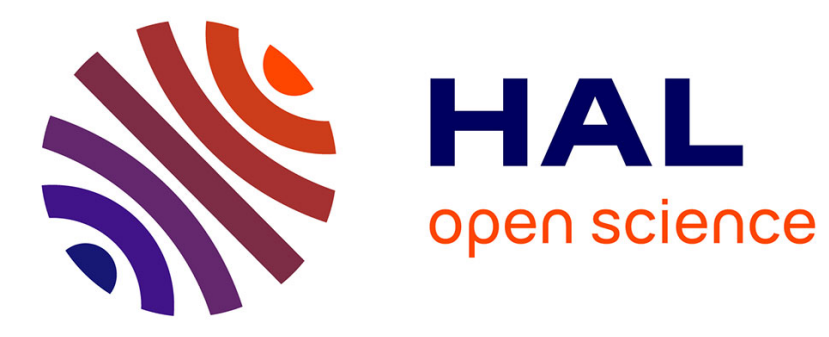

\title{
Haptic feedback tuning in colonoscopy simulation
}

Cédric Dumas, Timothy Coles, Hans de Visser, Caroline Gl Cao, Florian

Grimpen

\section{To cite this version:}

Cédric Dumas, Timothy Coles, Hans de Visser, Caroline Gl Cao, Florian Grimpen. Haptic feedback tuning in colonoscopy simulation. 2016 IEEE International Conference on Systems, Man, and Cybernetics (SMC), IEEE, Oct 2016, Budapest, Hungary. pp.4400 - 4404, 10.1109/SMC.2016.7844923 . hal-01495446

\section{HAL Id: hal-01495446 https://hal.science/hal-01495446}

Submitted on 25 Mar 2017

HAL is a multi-disciplinary open access archive for the deposit and dissemination of scientific research documents, whether they are published or not. The documents may come from teaching and research institutions in France or abroad, or from public or private research centers.
L'archive ouverte pluridisciplinaire HAL, est destinée au dépôt et à la diffusion de documents scientifiques de niveau recherche, publiés ou non, émanant des établissements d'enseignement et de recherche français ou étrangers, des laboratoires publics ou privés. 


\section{Haptic feedback tuning in colonoscopy simulation}

\author{
Cedric Dumas \\ Ecole des Mines de Nantes, IRCCyN \\ Nantes, France \\ Timothy Coles, Hans de Visser \\ AEHRC, CSIRO \\ Brisbane, Australia
}

\author{
Caroline GL Cao \\ EREL, Wright State University \\ Dayton, USA \\ Florian Grimpen \\ Royal Brisbane \& Women's Hospital \\ Brisbane, Australia
}

\begin{abstract}
According to medical experts, haptic realism is difficult to achieve, and even more difficult to have inter-expert agreement on the haptic feedback of one simulation. However haptic feedback is important in medical training, and allows educators to share the forces felt during a procedure if they know and trust what a particular virtual simulator will provide to the trainee. A new approach is proposed to refine bio-mechanical models with experts' input, to closely match the forces felt during a simulated procedure with an expert trainer's expectations. By allowing experts to tune a training scenario's haptic feedback as they trial a newly developed case, the experts can replicate their haptic perception and match their expectations with the simulation.
\end{abstract}

Keywords—Haptic; human factors; simulation; acceptability

\section{INTRODUCTION}

Modern surgery relies largely on laparoscopic, endoscopic or robotic-assisted techniques, where surgeons perform operations through a remote vision system (optical endoscopes or CCD cameras) using hand-held tools. In minimally invasive surgery (MIS), the manipulation of surgical tools relies, in large part, on image guidance with the feel of the tool deadened by tight fitting trocars. However, in highly delicate situations where precision and accuracy is critical for the success of procedures, the physician requires high haptic sensitivity. Unfortunately, kinesthetic perception is difficult to verbalize and share [1], and despite its importance, trainees rely on their own experiences to acquire motor skills, relying mainly on visual cues and practice, with no clear assessment or guidance provided.

Trainees can first practice with virtual simulators, but currently, according to medical experts, medical simulators in both research and educational settings lack in realistic haptic feedback [2]-[4], if haptics is available at all, including colonoscopy simulation [5].

Providing haptic (and visual) feedback in a surgical simulation requires intensive real-time computation to solve mechanical models and achieve realistic interactions between the user's tools and the virtual organs. Virtual haptic feedback is expensive to calculate and transmit, requiring complex mechanical feedback devices. While Finite Element Modelling (FEM) of surgical tool and organ interaction is possible, real time implementations are still very limited. Therefore, in current simulation solutions, virtual simulated force is limited to an approximation in both the model and force replication hardware.

Beyond these approximations necessary for real time simulation [6], the haptic quality experienced by the user is influenced by a number of other factors:

- the quality of the ex-vivo [7], [8] or in-vivo [9], [10] measurements of organ behavior and tissue compliance that inform the models

- the choice of haptic device and kind of tools simulated [11]

- the haptic sensitivity of the user [12]

- the complexity of the haptic perception process [13].

These factors mean it is likely that experts have different experiences and expectations when using a virtual simulation. Common points of expert contention are the lack of haptic realism, the inability to communicate haptics properly to the trainees, or not trusting the simulation.

One important advantage of virtual simulators is the ability to provide a wide range of clinical conditions for training [14]. Educators are able to configure training scenarios at will, and can change patient anatomy to vary the difficulty of a training session [6]. However, such control is limited to visual and anatomical changes and does not provide the same kind of configurable parameters for the haptic feedback, despite its importance in virtual surgical simulation [4].

We propose to improve the trustability of a simulator by providing a configurable haptic feedback to an expert: he will know what the simulator provides to the trainee; he will calibrate the simulator to its own perception and its own experience. So each trainer will have the opportunity to build his own training program, including visual and haptic configuration of every scenario.

This new approach proposes to use a self-tuning technique to modify the haptic feedback felt through an output device. Therefore, medical experts can act as their own encoder and decoder: providing their own input for haptic feedback while incorporating what they expect from a specific training 
scenario. The objective is to increase haptic feedback quality within simulations, and to increase the trustworthiness of the simulator as a training tool for experts and educators.

This study will evaluate this new approach by measuring how well gastroenterologists can tune haptic feedback provided by a simulator during a colonoscopy procedure. Loops in the colon are one of the most common difficulty in colonoscopy [15], and can result in loss of control of the endoscope as well as patient discomfort. Endoscope manipulation skills and haptic perception are critical skills to detect and reduce loops.

\section{PROTOCOL}

\section{A. Hypothesis}

We hypothesize that experts can dynamically tune haptic feedback to match their expectations during a simulated procedure, as medical experts have an accurate perception of the real procedure's haptic feedback [16], as the provided simulator has a highly realistic appearance and colon cases that can be configured. These configurable cases provide different levels of difficulty using a number of metrics, reducing the likelihood of a ceiling effect for experienced endoscopists [17].

In this study, experts are defined as those who have performed at least one hundred procedures per year during the last five years [18]. The effectiveness of allowing experts to tune a simulation will be evaluated by assessing:

- if experts choose to tune the simulator's force feedback when offered the chance

- if a force tuning consensus is reached by experts

- if the force can be tuned globally for the whole simulation

- the correlation of tuning between experts

- experts' satisfaction with the haptic feedback fidelity after the tuning.

An expert trainer's satisfaction with the haptic feedback will indicate their increased willingness to trust and therefore use the training simulation.

\section{B. Asuumptions}

The following experiment is based on two main assumptions. First, recruited participants are recognized as expert gastroenterologists [18], able to call on their experience to build an accurate representation of a procedure from a patient's complete profile. Second, participants have a good sense of the amount of force applied during a procedure, thanks to their experience and motor skills ability as endoscopists, and so can match a force using an adjustable haptic feedback device [19].

\section{Equipment}

The simulator (see figure 1) used in the experiment is a prototype colonoscopy simulator developed by the CSIRO in Australia [6]. It uses a custom haptic device developed by EPFL in Switzerland [25]. The haptic device supplies insertion and rotation information to the simulator at configurable rates (commonly 300-1000Hz), which in turn generates the physicsbased simulation of the interaction between a virtual endoscope and a virtual patient. The simulation creates both visual feedback, on a computer screen, and haptic feedback, via the haptic device, in longitudinal and rotation direction (ie. insertion force and torque). As the simulation needs to run in real-time, simplifications in the modelling of the interaction between endoscope and patient are inevitable. Hence, for a compelling training experience, tuning of the haptic feedback is essential. For this experiment, tuning will be limited to simple linear scaling of the calculated insertion force and torque to the force and torque generated by the haptic device. Observations show that colonoscopy, especially the critical withdrawal phase allowing a careful examination, is a slow motion activity. Then we consider the impact of velocity neglectable here. Separate scaling factors will be used for the force and the torque.
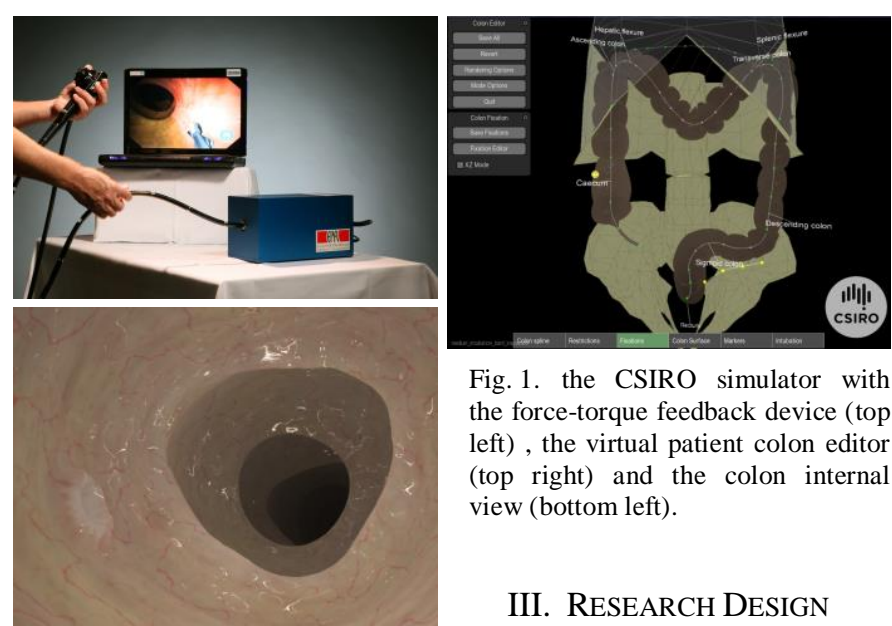

Fig. 1. the CSIRO simulator with the force-torque feedback device (top left), the virtual patient colon editor (top right) and the colon internal view (bottom left).

\section{RESEARCH DESIGN}

A group of gastroenterology experts from a local hospital is recruited for the study. Each participant performs a virtual colonoscopy on four virtual patients, with the ability to tune the haptic feedback. The study's ethics application has been approved by the Low Risk Review Panel of the CSIRO Animal and Human Ethics Committees under the reference number LR10/2013.

The colonoscopy (see figure 1) is to be performed using the CSIRO colonoscopy training simulator [6], equipped with an update of the EPFL designed force feedback haptic device [20]. The input device has two degrees of freedom, and provides one degree of force feedback and one degree of torque, up to $32 \mathrm{~N}$ translational (insertion of the endoscope) and $0.5 \mathrm{Nm}$ rotational (rotation of the endoscope).

Task. During each procedure subjects are asked to comment on and tune the haptic feedback according to a predetermined case difficulty and history of the virtual patient, while performing the insertion of the endoscope from the starting point of the case up to a visual landmark. The simulation output force can be increased online with two sliders operated by an assistant (while the subject can keep performing) - so only two parameters are tuned, the insertion force or the rotation torque of the endoscope, within the haptic device force / torque ranges. 
Data collection techniques. During simulated procedures the activity, forces exerted, endoscope position and velocity, as well as any haptic feedback adjustments made by the participant are recorded. A pre-questionnaire was used to determine the experience of each participant, including questions regarding years of practice and the number of colonoscopies performed each year. A post-questionnaire was used to measures the subjective experience of the gastroenterologist with the simulator.

Virtual scenario. Each participant performed endoscope insertions on four different virtual patients. The different exercises (insertion or rotation of the scope) were built with an expert gastroenterologist, who also tuned the simulator haptic baseline for those cases. The virtual patient cases were documented with a fake patient description containing medical history summarized as follow:

Case 1. 53 yo male presenting for screening colonoscopy on the basis of cancer family history, normal weight, no comorbidities

Case 2. 32 yo male presenting with bleeding, normal weight, no comorbidities

Case 3. 28 yo female with iron deficiency, normal weight, no comorbidities

Case 4. 65 yo female presented with abdominal pain and new onset constipation, BMI 27.

The subjects did not know the cases were built with increasing difficulty: the two first ones with a loop and an alpha loop happening when pushing in, the two last ones with an alpha loop already created in the sigmoid, where the subjects have first to detect there is a loop before unlooping, to be able to perform a proper insertion. Furthermore, the last one was proposed three times with 3 different starting points in the colon. The six cases (one case for each of the three first virtual patients, three cases with the last one) were difficult and have been built by an expert as challenging cases to be relevant in term of endoscope manipulation and haptic feedback information.

Data analysis. The forces applied during the colonoscopy procedure, and the difference between the simulator's biomechanical model calculation and the subject expectation were recorded.

Participants. A group of 9 physicians was recruited, 4 registrars with less than 5 years of experience but more than one hundred colonoscopy procedures performed, and 5 consultants with more than 5 years of experience and more than 500 hundred colonoscopy procedures performed in the last five years. We will keep those two groups separated (registrars and consultants) in the result section as we measured noticeable differences between them.

\section{RESUlts}

The original protocol was to ask the gastroenterologist to perform a complete virtual colonoscopy as they would do with the real patient. But this approach has encountered two strong limitations:
- From a human factor perspective, the gastroenterologist immerges himself in the simulated medical procedure, and performs his normal colonoscopy up to the secum, and then a withdrawal. He is struggling to provide haptic tuning during the whole simulated procedure, focusing on his task.

- From a technical perspective, with a new torque and force tuned along the colon, we can't invert our complicated biomechanical model of the colon to take the new forces automatically into account for a future simulation. It requires a manual re-parameterization of the biomechanical model to approximate the new values, a fastidious task.

The solution we found is to break the colonoscopy examination in smaller exercises to provide a part-task simulator: instead of performing a full virtual colonoscopy from the rectum to the secum, the expert is asked to perform the haptic tuning during an insertion of the endoscope in a limited portion of the colon (using the exact same simulator). The results are used to verify the hypothesis, but also to provide a haptic part-task trainer.

Pre-questionnaire. All the consultants and registrars agreed or strongly agreed simulation has an important role for learning to use equipment, learning fine motor skills and correct postures. Registrars agreed simulation has a role in replacing the apprenticeship method. Consequently, all participants felt involved with simulation and the proposed exercises.

All reported average IT skills and are not playing video games. One novice and one expert reported to have already use a virtual colonoscopy simulator, but long ago (two and four years ago). Consequently, all participants were novice in term of virtual simulation.

Post-questionnaires. On a scale from 1 (Not realistic) to 5 (Very Realistic), consultants rated 3,5 on 5 the force feedback quality and usefulness. The consultants rated 3,4 on 5 the graphic realism and the device handling realism compared to an actual colonoscopy with a patient.

On a scale from 1 (Not trustworthy) to 5 (Very trustworthy) to rate how the simulator is trustworthy to quantify accurate measures of performance, the simulator scored 3,6 with the consultants and 3,25 with the registrars.

Observations among the registrars. We observed a strong variation of skills among registrars. The first 3 subjects in the table below were able to stay in the middle of the lumen, detect the loops and unloop the colon, not getting lost often. The fourth novice was getting lost most of the time and was no able to detect the loops when he created them or when they were already created. The successful ones gave a positive feedback on the realism and sensitiveness of the device, but only the first one was able to tune the haptic feedback, the two others were not confident and acknowledged the provided forces. Finally, the last one explored a large range of forces, and succeeded to reach the visual landmark mainly through visual navigation. 
TABLE I. REGISTRARS RESULTS. RANGE IS THE RANGE OF EXPLORED FORCE (THE REGISTRAR DID NOT GIVE ANY FEEDBACK ON THE TORQUE), AN EMPTY CELL MEANS THEY WERE HAPPY WITH THE AMOUNT PROVIDED. FINAL

VALUE IS THE FINAL FORCE VARIATION THEY WERE HAPPY WITH. THE DURATION ROW GIVES AN INDICATION OF THE AMOUNT OF TIME SPEND FOR EACH CASE, 0 MEANS THEY DIDN'T HAD TIME TO COMPLETE.

\begin{tabular}{|c|c|c|c|c|c|c|c|}
\hline Novice & Case & 1 & 2 & 3 & 4.a & 4.b & 4.c \\
\hline \multirow{3}{*}{1} & Range (\%) & & $\begin{array}{ll}+1 & \text { to } \\
10 & \end{array}$ & $\begin{array}{l}+1 \text { to } \\
16\end{array}$ & & $\begin{array}{ll}+1 & \text { to } \\
10 & \end{array}$ & \\
\hline & Final value & $+0 \%$ & $+10 \%$ & $+15 \%$ & $+0 \%$ & $+10 \%$ & $+0 \%$ \\
\hline & Duration (s) & 568 & 400 & 281 & 196 & 256 & 587 \\
\hline \multirow{3}{*}{2} & Range (\%) & $\begin{array}{l}+1 \text { to } \\
10\end{array}$ & & & & & \\
\hline & Final value & $3 \%$ & $+0 \%$ & $+0 \%$ & $+0 \%$ & $+0 \%$ & \\
\hline & Duration (s) & 337 & 130 & 151 & 134 & 117 & 0 \\
\hline \multirow{3}{*}{3} & Range (\%) & $\begin{array}{ll}+1 & \text { to } \\
+3 & \end{array}$ & & & & & \\
\hline & Final value & $3 \%$ & $+0 \%$ & $+0 \%$ & $+0 \%$ & $+0 \%$ & $+0 \%$ \\
\hline & Duration (s) & 137 & 123 & 166 & 144 & 194 & 192 \\
\hline \multirow{3}{*}{4} & Range (\%) & $\begin{array}{ll}+1 & \text { to } \\
20 & \end{array}$ & $\begin{array}{l}+1 \text { to } \\
25\end{array}$ & $\begin{array}{ll}+1 & \text { to } \\
20 & \end{array}$ & $\begin{array}{l}+1 \text { to } \\
10\end{array}$ & & \\
\hline & Final value & $+20 \%$ & $+18 \%$ & $+15 \%$ & $+10 \%$ & $+0 \%$ & $+0 \%$ \\
\hline & Duration (s) & 703 & 215 & 299 & 567 & 359 & 357 \\
\hline
\end{tabular}

Observations among the consultants. The consultants spent nearly the same amount of time per case than the registrars (254 seconds/case versus 287 seconds/case). They provided more feedback with lots of talk-aloud during the exercises, but most of their comments were related to other dimensions of the simulator - useful to the simulator designers to improve the system, but not related to the present study as they were often happy with the haptic feedback provided for the different cases, being the torque or the force provided. All the experts detected the loops, when they created them or when they were already present at the beginning of the case.

TABLE II. CONSULTANTS RESULTS. RANGE IS THE EXPLORED VARIATION OF HAPTIC FEEDBACK. FINAL VALUE IS THE FORCE MODIFICATION CHOSEN BY THE SUBJECT. DURATION IS THE TIME SPENT ON THE CASE, 0 WHEN THE EVALUATION SESSION WAS INTERRUPTED BY HOSPITAL DUTIES.

\begin{tabular}{|c|c|c|c|c|c|c|c|}
\hline Consult. & Case & 1 & 2 & 3 & 4.a & 4.b & 4.c \\
\hline \multirow{3}{*}{1} & Range (\%) & & & & & & \\
\hline & Final value & $+0 \%$ & $+0 \%$ & $+0 \%$ & $+0 \%$ & $+0 \%$ & \\
\hline & Duration (s) & 366 & 79 & 80 & 166 & 126 & 0 \\
\hline \multirow{3}{*}{2} & Range (\%) & & & & & & \\
\hline & Final value & $+0 \%$ & $+0 \%$ & $+0 \%$ & $+0 \%$ & $+0 \%$ & $+0 \%$ \\
\hline & Duration (s) & 482 & 78 & 80 & 278 & 231 & 244 \\
\hline \multirow{3}{*}{3} & Range (\%) & & & $\begin{array}{l}+1 \text { to } \\
+10\end{array}$ & & & \\
\hline & Final value & $+0 \%$ & $+0 \%$ & $+10 \%$ & $+0 \%$ & $+0 \%$ & $+0 \%$ \\
\hline & Duration (s) & 274 & 168 & 544 & 638 & 607 & 335 \\
\hline \multirow{3}{*}{4} & Range (\%) & & & & & & \\
\hline & Final value & $+0 \%$ & $+0 \%$ & $+0 \%$ & $+0 \%$ & $+0 \%$ & \\
\hline & Duration (s) & 292 & 257 & 68 & 177 & 170 & 0 \\
\hline \multirow{3}{*}{5} & Range (\%) & $\begin{array}{l}+1 \text { to } \\
+11\end{array}$ & & & & & \\
\hline & Final value & $+10 \%$ & $+0 \%$ & $+0 \%$ & $+0 \%$ & $+0 \%$ & $+0 \%$ \\
\hline & Duration (s) & 481 & 123 & 95 & 142 & 175 & 377 \\
\hline
\end{tabular}

\section{ANALYSIS}

According to the expert tuning feedbacks (both registrars and consultants), the force feedback was consistent along the virtual colon, so the model was updated with a constant global force scaling. They did not required adjustments for specific anatomical parts. So the force can be tuned globally for the whole simulation when using the CSIRO endoscopic simulator.

The registrars were challenged by the difficult cases, and expressed their lack of confidence when asked about tuning the haptic feedback. They marked their psychological stress as moderate in the post-questionnaire, where consultants marked low. Those results are consistent with the difference of experience, and simulators (with more pedagogical cases) should help registrar building their skills faster and off-patients.

Consultants achieved a high inter-participant force agreement for each scenario, but without tuning the simulator's force feedback when offered the chance, as expected in the hypothesis. We interpret this by the deep involvement of the expert who first tuned the simulator - before the evaluation started, he spent 12 weeks with the simulator designers, at least 2 hours a week to evaluate the haptic performance as the developers were improving the behavior of the simulation.

It can be concluded that a force tuning consensus can be reached by experts, but this consensus can be achieved first by a fine tuning of a single expert with an accurate and long involvement in the model first tuning.

Even with this first tuning phase by a single expert, we believe the option of tuning the haptic (as the visual) feedback of the simulation for any expert is still important for the trustability of the simulator and its chance to be used later with confidence by the same expert for training novices: when the experts spent some time manipulating the simulator with difficult cases, their satisfaction with the haptic feedback fidelity will indicate their increased willingness to trust and therefore use the training simulation.

\section{CONCLUSION}

Medical simulation needs a high level of visual and haptic feedback fidelity to have a positive effect on training in colonoscopy. New methods need to be developed to overcome virtual simulator's haptic limitations - a new haptic selfparameterization of the training scenario is proposed: an interactive approach to elicit expert knowledge and haptic perception. After tuning a training scenario, the expert can be confident of the amount of force a trainee will experience, providing (asynchronous) haptic communication between him and the trainees.

The modification of our biomechanical model to match the expert tuning is a challenge as it is not invertible, like most models if they have not been designed on purpose. Tuned forces could be integrated into the original anatomical model by correcting the input parameters or the output force of the original biomechanical model. New cases can then use this updated force model to provide higher fidelity feedback from the outset. This iterative process to model development and refinement with medical experts' support will lead to more trusted simulation solutions, fueling wider acceptance.

This technique addresses the challenge of motor skills training for endoscopic procedures, and could be extended to other kind of simulation where experts can re-enact procedures. 


\section{REFERENCES}

[1] L. A. Jones and S. J. Lederman, Human Hand Function. Oxford University Press, 2006.

[2] C. Nezhat, "Surgical Therapies: Robotics and Endometriosis," in Endometriosis : science and practice, 2011, pp. 438-445.

[3] M. Schijven and J. Jakimowicz, "Construct Validity," Surg. Endosc. Interv. Tech., vol. 17, no. 5, pp. 803-810, May 2003.

[4] C. Vapenstad, E. F. Hofstad, T. Lango, R. Marvik, and M. K. Chmarra, "Perceiving Haptic Feedback in Virtual Reality Simulators," Surg. Endosc., vol. 27, no. 7, pp. 2391-2397, 2013.

[5] A. Hill, M. S. Horswill, A. M. Plooy, M. O. Watson, R. Karamatic, T. A. Basit, G. M. Wallis, S. Riek, R. Burgess-Limerick, and D. G. Hewett, "Assessing the realism of colonoscopy simulation: the development of an instrument and systematic comparison of 4 simulators," Gastrointest. Endosc., vol. 75, no. 3, pp. 631-640.e3, Mar. 2012.

[6] H. De Visser, J. Passenger, D. Conlan, C. Russ, D. Hellier, M. Cheng, O. Acosta, S. Ourselin, and O. Salvado, "DEVELOPING A NEXT GENERATION COLONOSCOPY SIMULATOR,” Int. J. Image Graph., vol. 10, no. 02, pp. 203-217, Apr. 2010.

[7] M. N. Appleyard, C. A. Mosse, T. N. Mills, G. D. Bell, F. D. Castillo, and C. P. Swain, "The measurement of forces exerted during colonoscopy," Gastrointest. Endosc., vol. 52, no. 2, pp. 237-240, Aug. 2000.

[8] R. Havre and O. H. Gilja, "Elastography and strain rate imaging of the gastrointestinal tract," Eur. J. Radiol.

[9] A. Samani, J. Bishop, C. Luginbuhl, and D. B. Plewes, "Measuring the elastic modulus of ex vivo small tissue samples," Phys. Med. Biol., vol. 48, no. 14, pp. 2183-2198, Jul. 2003.

[10] I. Brouwer, J. Ustin, L. Bentley, A. Sherman, N. Dhruv, and F. Tendick, "Measuring in vivo animal soft tissue properties for haptic modeling in surgical simulation," Stud. Health Technol. Inform., vol. 81, pp. 69-74, 2001.

[11] T. R. Coles, D. Meglan, and N. W. John, "The Role of Haptics in Medical Training Simulators: A Survey of the State of the Art," IEEE Trans. Haptics, vol. 4, no. 1, pp. 51-66, 2011.
[12] H. De Visser, K. Iyer, N. Good, and J. Passenger, "Torque Perception in Colonoscopy," in SimHealth 2013, Brisbane, Australia, 2013.

[13] J.-P. Bresciani, K. Drewing, and M. O. Ernst, "Human Haptic Perception and the Design of Haptic-Enhanced Virtual Environments," in The Sense of Touch and its Rendering, A. Bicchi, M. Buss, M. O. Ernst, and A. Peer, Eds. Springer Berlin Heidelberg, 2008, pp. 61-106.

[14] R. J. S. Md, V. T. O. Md, and S. B. I. Md, "Simulation Technology for Skills Training and Competency Assessment in Medical Education," J. Gen. Intern. Med., vol. 23, no. 1, pp. 46-49, Jan. 2008.

[15] T. N. Witte and R. Enns, "The difficult colonoscopy," Can. J. Gastroenterol., vol. 21, no. 8, pp. 487-490, Aug. 2007.

[16] D. G. Hewett, C. Zupanc, R. Burgess-Limerick, R. Karamatic, S. P. Riek, G. M. Wallis, A. M. Plooy, M. S. Horswill, A. Hill, and M. O. Watson, "M1428: A Colonoscopy Competency Framework Derived From Task Analysis," Gastrointest. Endosc., vol. 71, no. 5, p. AB218, Apr. 2010.

[17] D. G. Hewett, R. Burgess-Limerick, A. Hill, M. S. Horswill, A. M. Plooy, S. Riek, M. O. Watson, G. M. Wallis, and C. Zupanc, "Development and Evaluation of a National Colonoscopy Training Program,” University of Queensland, Jun. 2011.

[18] J. B. Marshall, "Technical proficiency of trainees performing colonoscopy: a learning curve," Gastrointest. Endosc., vol. 42, no. 4, pp. 287-291, Oct. 1995.

[19] D. Toffin, J. McIntyre, J. Droulez, A. Kemeny, and A. Berthoz, "Perception and Reproduction of Force Direction in the Horizontal Plane,” J. Neurophysiol., vol. 90, no. 5, pp. 3040-3053, 2003.

[20] E. Samur, L. Flaction, and H. Bleuler, "Design and Evaluation of a Novel Haptic Interface for Endoscopic Simulation," IEEE Trans. Haptics, vol. 5, no. 4, pp. 301-311, 2012. 